\title{
Single-Grain Germanium TFTs
}

\author{
Ryoichi Ishihara, Tao Chen, A. Baiano, M.R. Tajari Mofrad and C.I.M Beenakker \\ Delft University of Technology, Delft Institute of Microsystems and Nanoelectronics \\ (DIMES), Delft, The Netherlands
}

\begin{abstract}
We review our recent achievements in location-control of $\mathrm{Ge}$ grains and high performance single-grain (SG) Ge thin film transistor (TFT) fabricated inside a Ge grain. Large Ge grains having a grain size of $10 \mu \mathrm{m}$ were obtained at predetermined positions by the $\mu$-Czochralski process using excimer-laser and sputtered a-Ge layer. TFTs were fabricated inside the single grain of Ge. Capping silicon dioxide was applied before the laser crystallization with which a high quality Ge/insulator interface was formed during the laser annealing. Source/drain regions were formed by doped $\mathrm{Si}$ instead of the Ge, which ensures a low contact resistance and suppresses fast dopant diffusion in the channel. Nand p-channel SG Ge TFTs showed electron and hole mobility of as high as $3337 \mathrm{~cm}^{2} / \mathrm{Vs}$ and $1719 \mathrm{~cm}^{2} / \mathrm{Vs}$, respectively. On/off current ratio for the both types of TFTs was in the order of $10^{7}$.
\end{abstract}

\section{Introduction}

Germanium (Ge) has long been known to possess superior electron and hole mobilities than silicon ( $\mathrm{Si}$ ). [1] The large electron mobilities are highly desirable for high speed electronic circuits based on both ULSI and TFT technologies. In addition, Ge can absorb light with a longer wavelength because of the narrow band-gap. Therefore a near infrared photo-diode is expected to be realized for use as optical interconnect in future ULSI. Therefore both MOSFET and TFT using Ge have been recently studied extensively.

In the Ge MOSFET, there are several issues in the device fabrication point of view. First problem is the high interface states density between the Ge and an insulator which lowers carrier mobility [2]. Second problem is the fast dopant diffusion into channel during impurity annealing, which shortens the channel and increases the off-current.

In the TFTs using Ge channel, device has been fabricated with polycrystalline-Ge on a glass substrate. Because the film is polycrystalline, mobility becomes even lower by scattering at grain boundaries [3]. Off current becomes also very large due to carrier generation at the boundaries, resulting in very small on/off current ratio.

Those problems will be solved if the transistor can be made inside a single grain of Ge with a new device process realizing high quality Ge/gate insulator interface and negligible doping diffusion into the channel. Here, accurate 2D location control of large Ge grains is essential to ensure uniform property. For $\mathrm{Si}$, we have reported that location of individual Si grain can be controlled at predetermined position by a developed process so called $\mu$-Czochralski process [4,5] based on pulsed-laser crystallization. Grain size of the Si grains can be up to $9 \mu \mathrm{m}$ and TFTs fabricated inside the single Si grain show high 
carrier mobilities for electron and holes $\left(600 \mathrm{~cm}^{2} / \mathrm{Vs}\right.$ and $\left.250 \mathrm{~cm}^{2} / \mathrm{Vs}\right)$, despite of the lowtemperature $\left(<350^{\circ} \mathrm{C}\right)$ process [6]. We have also demonstrated RF circuit with low-noise amplifier operating at $440 \mathrm{MHz}$ [7] and monolithic 3D-IC with CMOS inverters with stacked two SG-TFTs [8]. If the process is applied to Ge, we expect even better electrical properties owing to the high carrier mobilities.

In this paper we will review our recent achievements in location-control of Ge grains and high performance single-grain (SG) Ge thin film transistor (TFT) fabricated inside the Ge grain. Large Ge grains having a maximum grain size of as large as $10 \mu \mathrm{m}$ were obtained at predetermined positions using excimer-laser. A high quality $\mathrm{Ge} /$ insulator interface was formed with a capping silicon dioxide which was annealed during the laser crystallization. TFTs were fabricated inside the single Ge grains with source and drain made with doped-Si. N- and p-channel SG Ge TFTs showed electron and hole mobility of $3337 \mathrm{~cm}^{2} / \mathrm{Vs}$ and $1719 \mathrm{~cm}^{2} / \mathrm{Vs}$, respectively. On/off current ratio for the both types of TFTs was in the order of $10^{7}$.

\section{LOCATION CONTROL OF GERMANIUM GRAINS}

We first discuss 2D location control of germanium grains with the $\mu$-Czochralski process based on excimer-laser crystallization.

\section{The $\mu$-Czochralski (grain-filter) process with germanium}

We have proposed the $\mu$-Czochralski process for 2D location control of semiconductor grains. As depicted in Fig. 1(a), the $\mu$-Czochralski process has a locally increased thickness of the semiconductor film in a cavity (grain-filter) in a substrate. Upon excimer-laser irradiation, the semiconductor film surrounding the grain-filter melts completely, whereas the grain filter won't melt completely due to the large heat dissipation and heat capacitance there. During vertical growth of pre-existing seeds in the grain filter, occlusion of grains occurs reducing the number of growing grains. By increasing the aspect ratio of more than about 7 , only single grain can be filtered out from the many pre-existing fine grains. In case of $\mathrm{Si}$, we have demonstrated that, using a 250 $\mathrm{nm}$ thick LPCVD a-Si film as a precursor, a single grain of Si with a size of up to $9 \mu \mathrm{m}$ can be positioned on top of the grain-filter. [4,5] We applied the method for 2D location control of Ge grains.

\section{Experimental}

2D location control of germanium grains are obtained as follows [9]. Firstly, $1 \mu \mathrm{m}$ diameter holes are formed in oxide and $840 \mathrm{~nm}$ thick $\mathrm{SiO}_{2}$ layer is then $2 \mathrm{nd} \mathrm{SiO}_{2}$ was deposited by TEOS-PECVD at a substrate temperature of $350{ }^{\circ} \mathrm{C}$ in order to reduce the grain-filter diameter to approximately $100 \mathrm{~nm}$. An etch-back of the $\mathrm{SiO}_{2}$ is needed to make slope of sidewall of the grain-filter gentle for the following sputtering deposition of $250 \mathrm{~nm}$ thick a-Ge film at different substrate temperatures of $100{ }^{\circ} \mathrm{C}, 400{ }^{\circ} \mathrm{C}$, and $550^{\circ} \mathrm{C}$. Sputtering is performed with pulsed DC mode with a power of $1 \mathrm{~kW}$, frequency of 75 $\mathrm{kHz}$, pulse with of $496 \mathrm{~ns}$ and duty cycle of 3.7 percent. 
Then a single pulse of $\mathrm{XeCl}(308 \mathrm{~nm}, 25 \mathrm{~ns})$ excimer laser irradiates the Ge surface with energy densities ranging from 200 up to $800 \mathrm{~mJ} / \mathrm{cm}^{2}$. Figure 1(b) shows an example of SEM image of location-controlled Ge grain with a-Ge deposited at $550^{\circ} \mathrm{C}$ [9]. It can be seen that Ge grains having almost squared shape with a side length of $6 \mu \mathrm{m}$ are successfully positioned on the grain filters. It can be also noticed that the surface inside the germanium grain is flat despite the gentle slope of the holes and hence initial indentation of Ge surface there. This is because the melting of the a-Ge by the laser pulse induced mass transfer into the grain filter and flattened the surface.

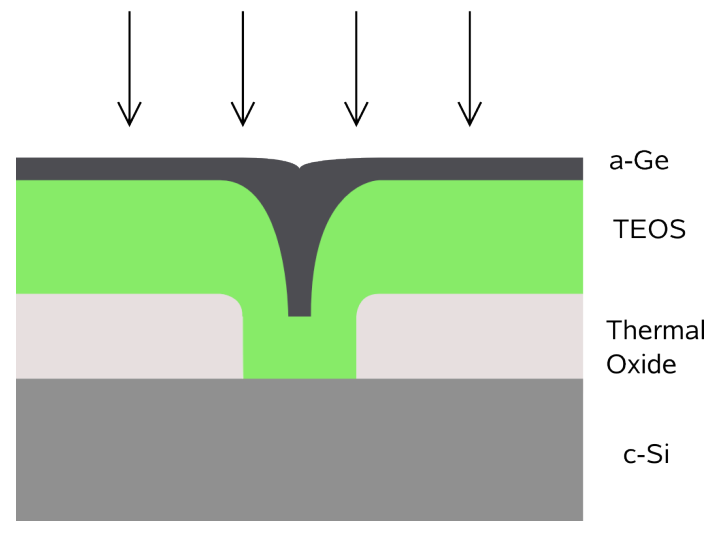

(a)

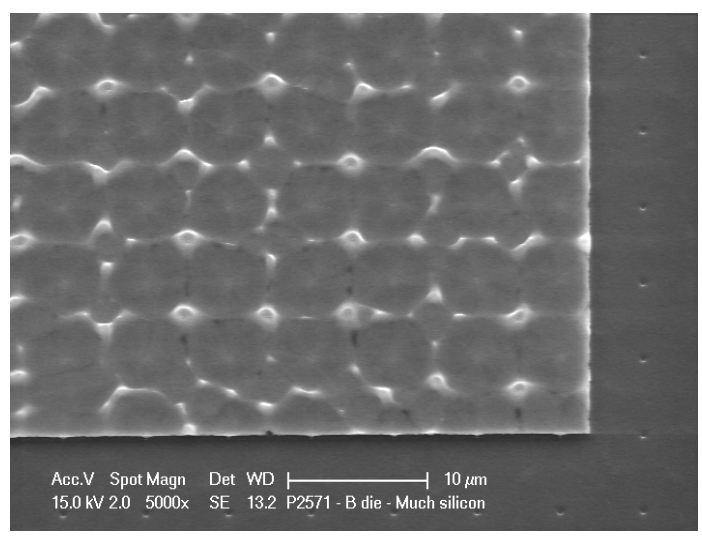

(b)

Figure 1. Schematic diagram (a) and SEM image (b) of location-controlled germanium grains by the $\mu$-Czochralski process. Pitch of the grain-filters is $6 \mu \mathrm{m}$.

\section{Grain size}

Figure 3 shows grain size of the location-controlled Ge as a function of energy densities of the excimer-laser. In the figure, as a reference, grain size of Si deposited with LPCVD with the same thickness is also plotted. For the both $\mathrm{Ge}$ and $\mathrm{Si}$, the grain sizes increase with the energy density. Difference between the two is however that the location control of Ge grains is obtained by much lower energy range than that of the Si. This is

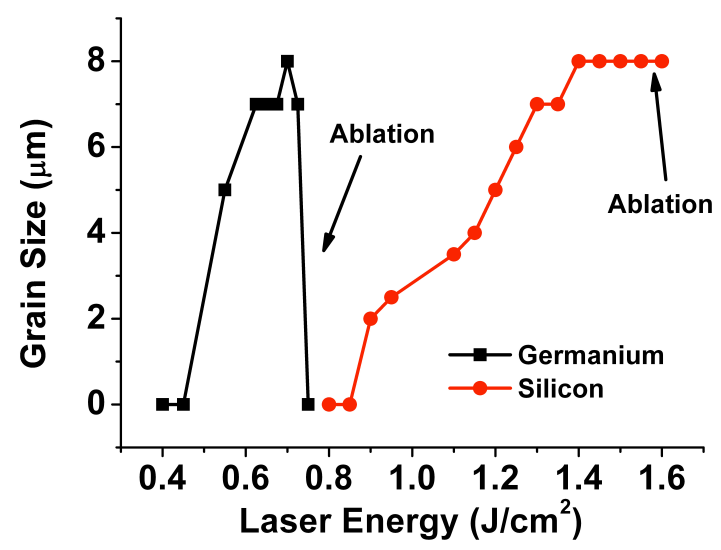

Figure 2. Grain size of location-controlled Ge (sputtered at $550^{\circ} \mathrm{C}$ ) and $\mathrm{Si}$ (LPCVD at $545^{\circ} \mathrm{C}$ ) versus laser energy density 
consistent with the lower melting temperature of the Ge than that of the Si. Maximum grain size of $\mathrm{Ge}$ is about $7 \mu \mathrm{m}$, which is slightly smaller than that of $\mathrm{Si}(8 \mu \mathrm{m})$. After reaching the maximum grain size, it drops dramatically to the small value of about a few hundred nano meters. In this regime where the grain filter seems to be completely melted, many of those small grains are uniformly grown over the surface and there is no evident location-control of the grains observed. Such behavior is notably different than the case of Si, where still large grains are grown on the grain filter even after complete melting the grain filter and reaching the maximum grain size. [5] Although exact reason for this difference is still under investigation, it indicates clearly difference in nucleation behavior between the molten-Ge and molten-Si. For both materials, the film gets agglomerated with an excess energy density.

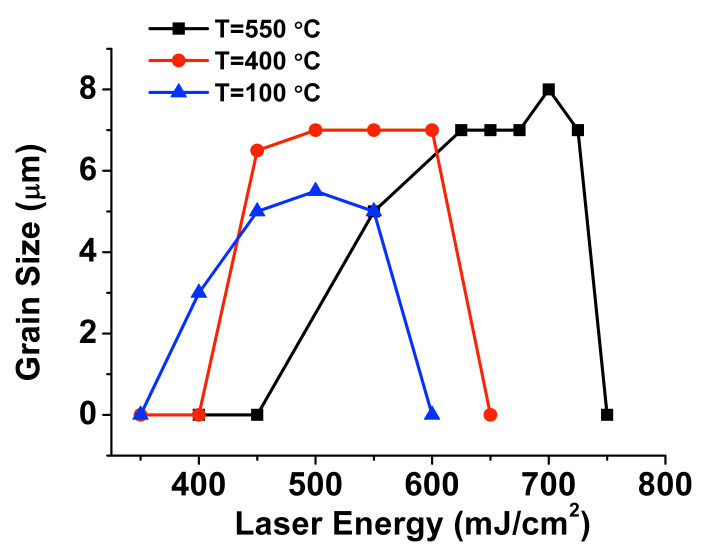

Figure 3. Grain size of location-controlled Ge for various deposition temperature

We have performed similar experiment with a-Ge sputtered at different temperature $\mathrm{T}$. As shown in the Fig. 3, with decreasing the temperature of the sputtering, the onset of the grain size enlargement occurs at lower energy densities. For $\mathrm{T}$ of $400{ }^{\circ} \mathrm{C}$, the process window becomes slightly smaller $\left(450-600 \mathrm{~mJ} / \mathrm{cm}^{2}\right)$ and the grain size can reach $7 \mu \mathrm{m}$ at most. For $\mathrm{T}$ of $100{ }^{\circ} \mathrm{C}$, the maximum grain size decreased to $5 \mu \mathrm{m}$. After reaching the maximum grain size, the behavior is the same for all temperature; namely, the sudden drop of grain size and the random formation of the grains, followed by the ablation at an excess energy.

The maximum grain size was further increased by elongating the pulse duration. With a longer pulse duration of $180 \mathrm{~ns}$, as shown in the Fig. 4 (a), the grain size can reach 10 $\mu \mathrm{m}$, which is even larger than that of Si deposited by LPCVD $(10 \mu \mathrm{m})$. The enlargement of grain by the longer pulse duration is explained by the increased amount of heat deposited directly under the germanium due to the long diffusion time and onset delay in homogeneous nucleation in the molten-Ge, which stops the lateral grain growth from the grain filter and eventually limits the maximum grain size.

Crystal quality and residual stress in the Ge grain were measured by micro-Raman spectroscopy. As shown in Fig. 4(b), the Raman shift peak of SG-Ge is at a lower wave number than that of Ge bulk by $3.812 \mathrm{~cm}^{-1}$ which suggests high tensile stress is formed in the SG-Ge during ELC because of the large difference in the heat expansion coefficient. 


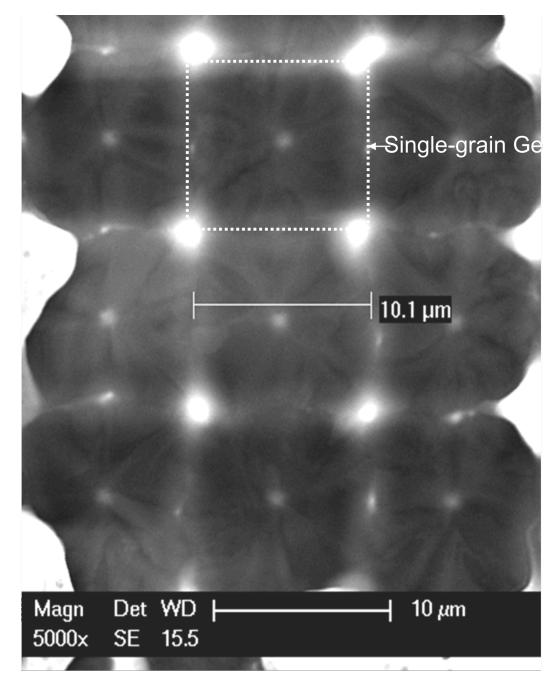

(a)

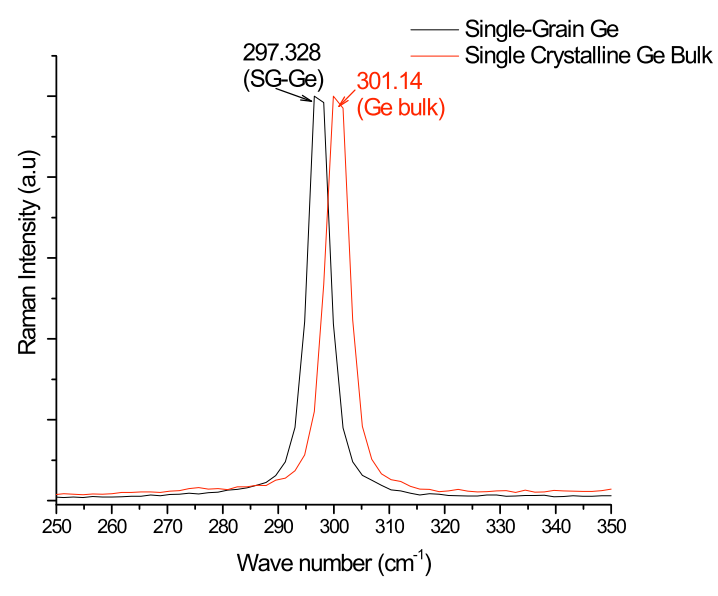

(b)

Figure 4. SEM (a) and Raman shift (b) of location-controlled Ge grains obtained with a longer pulse duration of 180ns.

\section{Microstructure of germanium grains}

We have analyzed microstructure of the obtained location-controlled Ge grain with the electron backscattering diffraction (EBSD). As shown from the Fig. 5(a), the planar defects generating from the grain filter holes are only coincident site lattice (CSL) with meanly $\Sigma 3$ and $\Sigma 9$, whereas random grain boundaries are only on the edge of the grain where others grains collide. Similar result has been obtained for Si as well. [10] Electrical activities of the CLSs must be negligible as in the case of Si. The quality of the single crystal of Ge depends on the grain filter diameter. In fact, as shown in Fig. 5(b), for the large diameter of $500 \mathrm{~nm}$ of the grain filter, random grain boundaries are incorporated in the location-controlled grains due to decreased filtering of grains during vertical growth in the grain filter. Similar trend has been observed for the case of Si. [10]

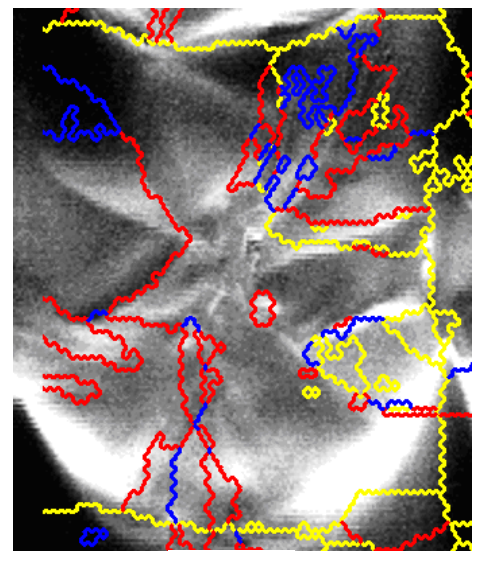

(a)

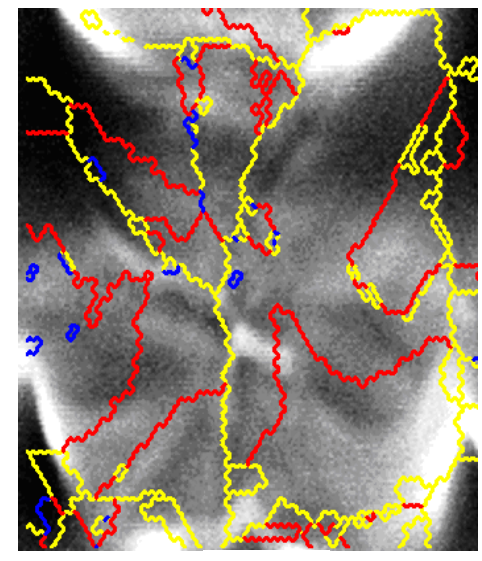

(b)

Figure 5. Electron backscattering diffraction (EBSD) using grain filter size of $100 \mathrm{~nm}$ (a) and $500 \mathrm{~nm}$ (b). The yellow lines are random grain boundaries, the red: $\Sigma 3 \mathrm{CSL}$ boundary and the blue: $\Sigma 9$ CSL boundaries. 


\section{Single-grain germanium TFTs}

In this section we propose a new device structure for Ge FETs and review fabrication process and electrical properties of single-grain Ge TFT made inside a Ge grain.

\section{$\underline{\text { Single-grain Ge TFTs }}$}

In the Ge MOSFET, device is fabricated in germanium on insulator (GOI) substrate because of advantage of low RF loss. Apart from difficulty in obtaining defect free GOI substrate, there are several issues in the device fabrication point of view. First problem is the high interface states density between the Ge and an insulator which degrade subthreshold swing and lowers carrier mobility. [2] The traps are created due to desorption of $\mathrm{GeO}$ [11-13], which is native germanium oxide and unstable chemically and thermally.

Second problem in the Ge transistor is about doping in the source and drain. Because of the much lower solubility limit of Ge than that of $\mathrm{Si}$, contact resistance with a metal and parasitic resistance in the source and drain region become very high, resulting in low on-current. Also because of the same reason, the dopants diffuse easily into the channel, which leads to high leakage current. In addition, the dopants in Ge need to be annealed at higher temperature than that for $\mathrm{Si}$ to restore crystal damage due to implantation and interstitials made during the process.

To overcome those problems we propose a new device process and structure of Ge transistor. [16] As shown in Fig. 6, the device has a thin $\mathrm{SiO}_{2}$ layer as gate insulator which is deposited at a low-temperature before laser crystallization of Ge. Upon irradiation of a pulsed-laser, the Ge surface absorb the light and heats up and eventually melts the film completely. (If there is grain-filter underneath, the grain-filter won't be melted.) The surface $\mathrm{GeO}$ is also completely melted, however it would not be desorpted and will be kept at the interface because of the presence of the capping $\mathrm{SiO}_{2}$. After turning off the laser pulse, the molten-Ge starts solidifying. At the end of the solidification of $\mathrm{Ge}$, we expect that an atomically thin, high quality $\mathrm{GeO}_{2}$ layer $[14,15]$ grows at the interface of $\mathrm{Ge} / \mathrm{SiO}_{2}$ by the presence molten $\mathrm{GeO}$ and oxygen atoms in the $\mathrm{SiO}_{2}$. During the whole crystallization process of $\mathrm{Ge}$, the $\mathrm{SiO}_{2}$ is annealed by the heat and the bulk property is also improved.

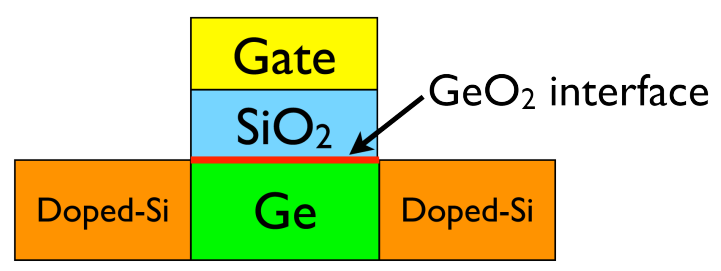

Figure 6. Proposed structure of the single-grain germanium TFTs. 
The device is equipped with doped-Si for the source and drain regions. The dopants are implanted by an ion-implantation and activated by a pulsed-laser. The high solubility of Si ensures a low contact resistance to a metal and also a low source and drain parasitic resistance. The high solubility also prevents dopant diffusion into the channel. The pulsed-laser annealing effectively activate the dopants in a very short time without causing unwanted diffusion of dopants into the channel region.

\section{$\underline{\text { Fabrication process }}$}

Figure. 7 (a)-(f) show schematic cross section of fabricated device [16]. Locationcontrol of Ge grains is performed basically the same procedures as the aforementioned process. In this process, before depositing a-Ge into the $100 \mathrm{~nm}$ diameter grain-filter, in order to control the crystal orientation of germanium, we first prepared (001) oriented Si seed inside the grain filter hole to control the orientation of the Ge grains. The textured seed was fabricated by MILC (metal induced lateral crystallization) which has been reported in our earlier paper [16] (Fig. 7(a)-(b)). CMP polished a way the Si film leaving only the seed of (001) oriented Si inside the grain filters. Then we have deposited a $250 \mathrm{~nm}$ thick a-Ge layer by sputtering at a substrate temperature of $450^{\circ} \mathrm{C}$. Then a $50 \mathrm{~nm}$ thick $\mathrm{SiO}_{2}$ layer was deposited by TEOS-PECVD at $350^{\circ} \mathrm{C}$, as a capping layer for the following excimer laser crystallization (Fig.7(c)). The sample was crystallized by excimer-laser $(\lambda=308 \mathrm{~nm}, \mathrm{t}=180 \mathrm{~ns})$ with a substrate temperature of $450^{\circ} \mathrm{C}$ (Fig.7(d)). During the laser crystallization, Ge grains grew epitaxially from the (001) oriented $\mathrm{Si}$ seed (Fig. 7(c)). After patterning the $\mathrm{Ge}$ and the capping $\mathrm{SiO}_{2}$ into islands, additional $40 \mathrm{~nm} \mathrm{SiO} 2$ was deposited by the TEOS PECVD. The total thickness of the gate oxide is

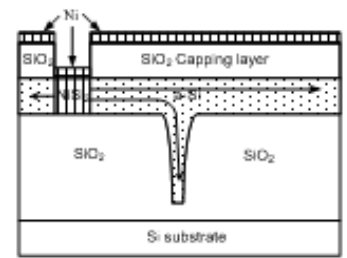

(a)

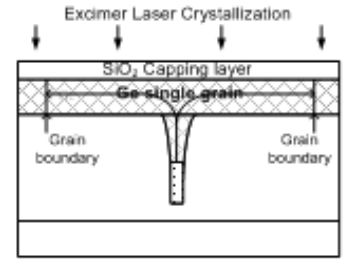

(c)

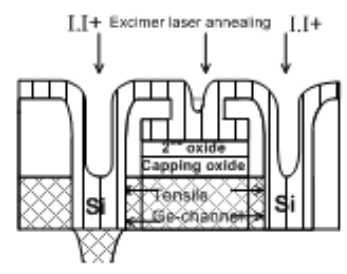

(e)

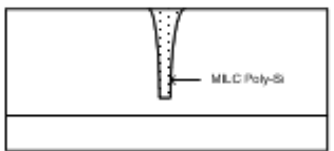

(b)

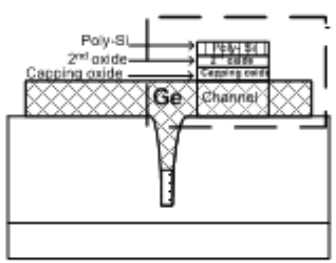

(d)

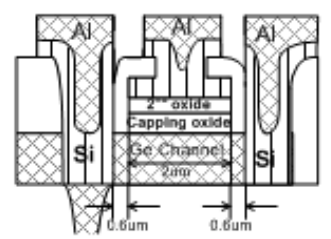

(f)

Figure 7. Schematics of fabrication process of the single-grain Ge TFTs 
90nm. Then a $250 \mathrm{~nm}$ thick a-Si was deposited as poly-gate by LPCVD at $545^{\circ} \mathrm{C}$ and phosphorous and boron ions were implanted with $10^{16}$ ions $/ \mathrm{cm}^{2}$ for $\mathrm{n}$ - and $\mathrm{p}$-channel TFTs, respectively (Fig.7(d)). Then the dopants were activated by excimer laser annealing (ELA) with energy density of $300 \mathrm{~mJ} / \mathrm{cm}^{2}$ at room temperature. The poly-Si and the gate $\mathrm{SiO}_{2}$ were etched with a gate mask. After deposition of a passivation $\mathrm{SiO}_{2}$, contact hole for source and drain was opened. The Ge in the source/drain contact hole was etched away by anisotropic plasma etching, leaving a $0.6 \mu \mathrm{m}$ offset of intrinsic Ge adjacent to the channel (Fig.7(e)). A 250nm thick a-Si was deposited as source/drain by LPCVD and the same implantation and annealing scheme was repeated (Fig.7(e)). The channel width and length was $2 \mu \mathrm{m}$ for both p- and n-channel TFTs. After deposition and patterning $\mathrm{Al}$ pads, the sample was annealed at $400^{\circ} \mathrm{C}$ in $\mathrm{H}_{2}$ (Fig.7(f)).

\section{TFT characterization}

Figure 8 shows transfer characteristics of the single-grain (SG) Ge TFTs. Effective mobility was calculated by transconductance $\left(\mathrm{g}_{\mathrm{m}}\right)$ at Vth. For n-channel, the SG Ge TFT shows an effective mobility of $3337 \mathrm{~cm}^{2} / \mathrm{Vs}$ with a large on/off ratio of $10^{8}$ at $\mathrm{V}_{\mathrm{DS}}=0.1 \mathrm{~V}$. For p-channel, SG Ge TFT shows an effective mobility of $1719 \mathrm{~cm}^{2} / \mathrm{Vs}$ with a large on/ off ratio of $10^{7}$ at $\mathrm{V}_{\mathrm{DS}}=0.05 \mathrm{~V}$. The $\mathrm{S}$ value for $\mathrm{n}$ - and $\mathrm{p}$-channel $\mathrm{SG}$ TFT is $0.19 / \mathrm{dec}$. and $0.17 \mathrm{~V} /$ dec., respectively. The high mobility and low $\mathrm{S}$ value suggest that a good interface property between $\mathrm{Ge}$ and gate $\mathrm{SiO}_{2}$ was achieved due to the thin $\mathrm{GeO}_{2}$ interface layer. The off-current was $10 \mathrm{pA}$ and $0.1 \mathrm{pA}$ for $\mathrm{n}$ - and $\mathrm{p}$-channel, respectively, which suggest that phosphorous diffusion into Ge is effectively suppressed by the Ge offset and the pulsed-laser activation.
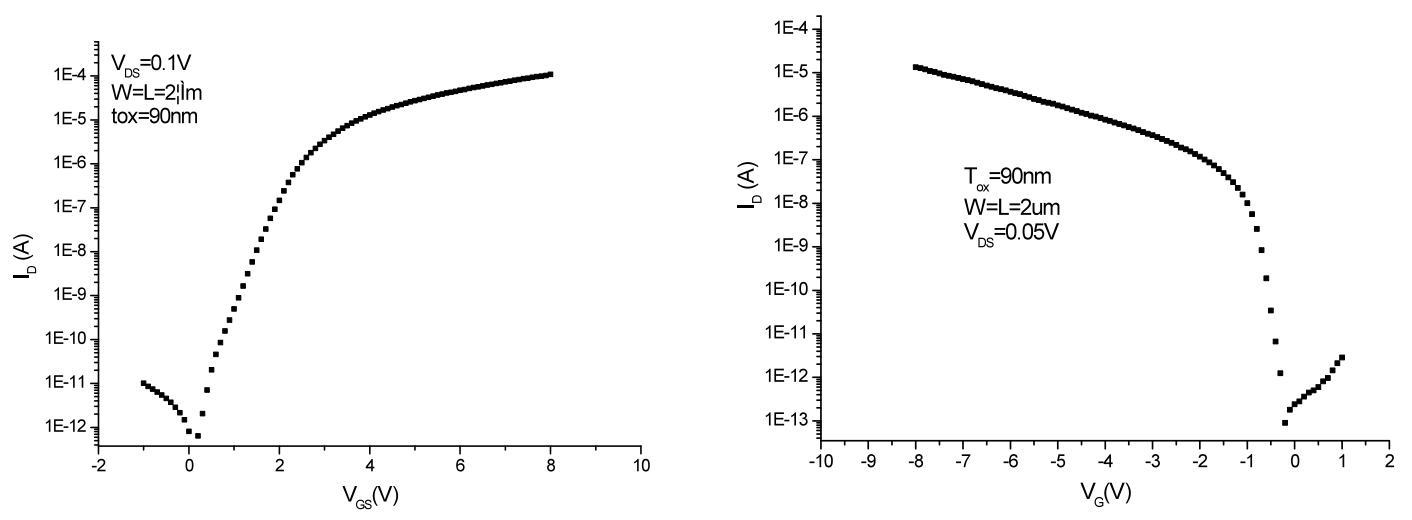

Figure 8. Transfer characteristics of $n-$ (a) and p-channel (b) single-grain germanium TFTs

Figure 9 shows output characteristic of n- and p-channel devices. For the both types of devices, the current increases exponentially. Similar property has been observed for the tunneling MOSFETs. In addition the current does not saturate in, at least, the range of the

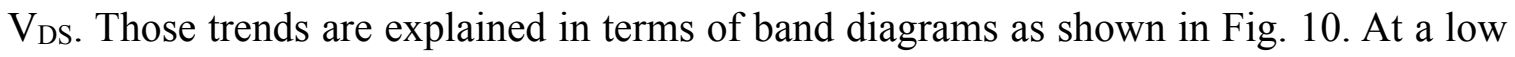
drain bias $\mathrm{V}_{\mathrm{DS}}$, with increasing the gate bias $\mathrm{V}_{\mathrm{GS}}$, the source barrier between Ge channel and doped-Si becomes so low that the electrons can emit through the intrinsic Ge barrier into the channel (Fig.10 (b)). The electron flow is however hindered by potential barrier 
at the Ge offset at drain side. By increasing the $\mathrm{V}_{\mathrm{GS}}$ further the electrons are collected at the drain region due to the lowered barrier at the Ge offset at drain (Fig. 10(c)). With increasing the $\mathrm{V}_{\mathrm{DS}}$, the barriers at the intrinsic Ge offset at source and drain become so low that a lot of electrons can emit into the channel from the source and collected by the drain which result in very high drain current (Fig.10 (e)).
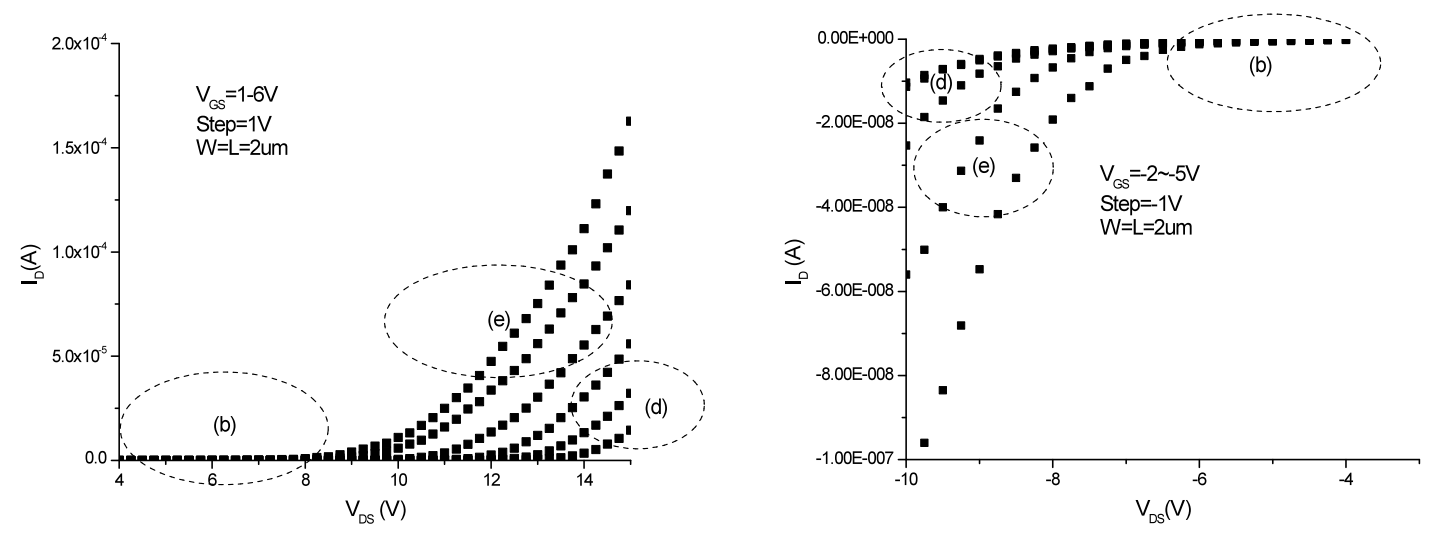

Figure 9. Output characteristics of n- (a) and p-channel (b) single-grain germanium TFTs

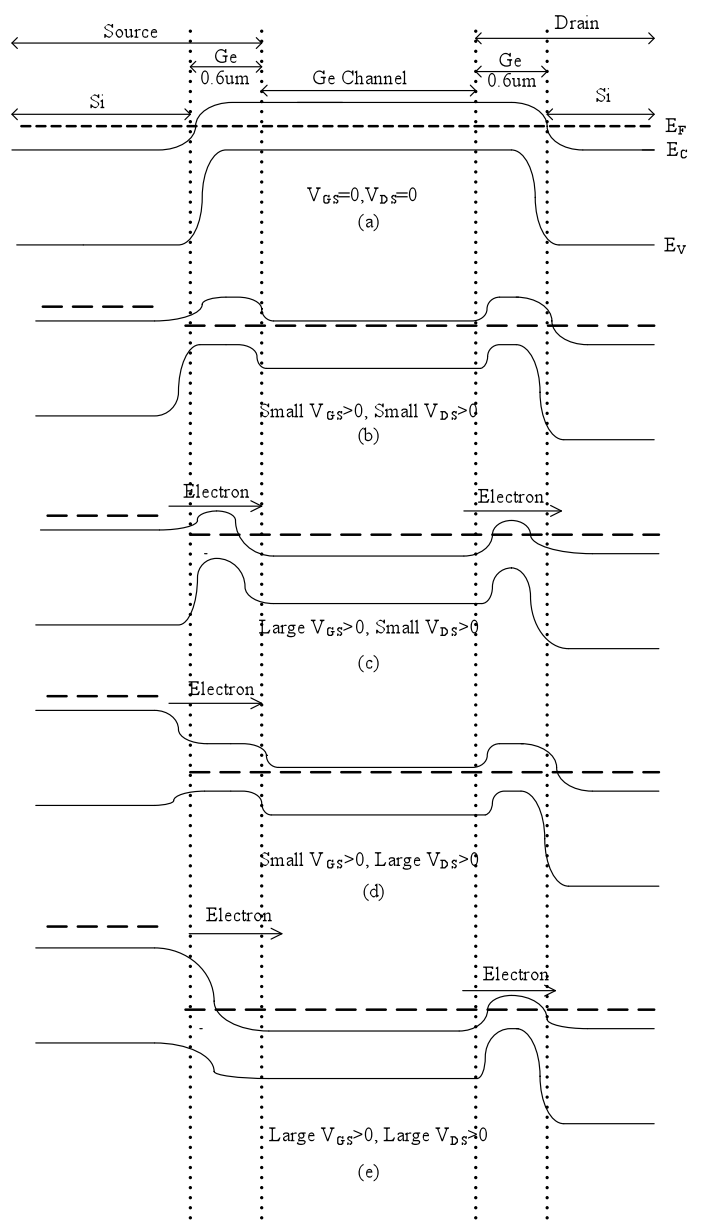

Figure 10. Band diagram of the single-grain Ge TFTs explaining possible transport mechanism of the TFTs with bias conditions of $\mathrm{V}_{\mathrm{GS}}=\mathrm{V}_{\mathrm{DS}}=0$ (a), low $\mathrm{V}_{\mathrm{DS}}$ with low (b) and high $\mathrm{V}_{\mathrm{GS}}(\mathrm{c})$ and high $\mathrm{V}_{\mathrm{DS}}$ with low (d) and high $\mathrm{V}_{\mathrm{GS}}(\mathrm{e})$. 


\section{Conclusions}

We reviewed our recent achievements in location-control of Ge grains and high performance single-grain (SG) Ge thin film transistor (TFT) fabricated inside the Ge grain. Large Ge grains having a grain size of $10 \mu \mathrm{m}$ were obtained at predetermined positions by the $\mu$-Czochralski process using excimer-laser and sputtered Ge layer. TFTs were fabricated inside the single grain of Ge. Capping silicon dioxide was applied before the laser crystallization with which a high quality Ge/insulator interface was formed during the laser crystallization process. Source and drain were formed by doped Si instead of the Ge counterpart, which ensure a low contact resistance and suppresses fast dopant diffusion in the channel. N- and p-channel SG Ge TFTs showed electron and hole mobility of as high as $3337 \mathrm{~cm}^{2} / \mathrm{Vs}$ and $1719 \mathrm{~cm}^{2} / \mathrm{Vs}$, respectively. On/off current ratio for the both types of TFTs was in the order of $10^{7}$. This will open several new applications in TFTs for displays with ultra-wide-band RF wireless communication interface and monolithic 3D-ICs with optical interconnect.

\section{Acknowledgments}

The authors would like to thank the Dutch Technology Foundation STW for their financial support and the cleanroom staff of DIMES technology center for their support during the fabrication of these devices.

\section{References}

1. R. H. Reuss, et al., Proceeding of IEEE, vol. 93, no. 7, (2005)

2. K. Saraswat, et al., IEDM 2006, 659.

3. T. Sadoh, et al., Jpn. J. Appl. Phy., 46, 3B, (2007) 1250

4. R. Ishihara, et al., Proc. SPIE, 4295, 14 (2001)

5. R. Ishihara, et al., Solid State Electronics, 52, 353-358 (2008)

6. V. Rana, et al., IEEE Trans. Electron Devices, 52, 12, 2622 (2005)

7. N. Saputra, et al., J. Solid State Circuits, 43, 7, 1563-1576 (2008)

8. M.R.T. Mofrad, et al., Japanese Journal of Applied Physics, vol. 48, (2009)

9. A. Baiano et al., Trans. ECS, 16(9), 153, (2008).

10. R. Ishihara, et al., J. Crystal Growth, 299, 2, 316 (2007)

11. E.P. Gusev, et al., Appl. Phy. Lett., 85, 2334. (2004)

12. C.O. Chui, et al., IEEE Electron Device Lett., 23, 473 (2002)

13. D.S.Yu, et al., IEEE Electron Device Lett., 25, 559 (2004)

14. D. Kuzum, et al., IEEE Electron Device Lett., 29 (4) (2008) 328-330

15. C.H.Lee, et al., Appl. Phys. Express 2 (2009) 071404

16. T. Chen, et al., IEEE Trans. Electron Devices, 58, p. 223 (2010) 